\title{
Computed tomography in diagnosis and management of aneurysm of the vein of Galen
}

\author{
P. MA CPHERSON, G. M. TEAS D A L A A D K. W L INDSAY \\ From the Departments of Neuroradiology and Neurosurgery, Institute of Neurological Sciences, \\ Southern General Hospital, Glasgow
}

SUMMARY A case of aneurysm of the vein of Galen is presented to demonstrate the value of $\stackrel{\mathbb{D}}{\triangle}$ computed tomography, not only as a diagnostic procedure, but also in the overall management of the condition.

Most cases of vein of Galen aneurysm have been diagnosed at necropsy or by arteriography. A presumptive diagnosis can be made from the presence of curvilinear calcification on the skull film (Amacher and Shillito, 1973), from lumbar pneumoencephalography (Morris, 1971), or ventriculography although the last investigation may be misleading (Hirano and Terry, 1958). Berger et al. (1976), Milhorat (1978), and Weisberg et al. (1978) state that with computed tomography it has become possible to demonstrate a mass above and behind the third ventricle but that the definitive diagnosis of a vein of Galen aneurysm is made by angiography.

It has been suggested that all arteriovenous malformations involving the Galenic system should be grouped under one term, noting whether the arteriovenous fistula was adjacent or remote (O'Brien and Schechter, 1970). However, Amacher and Shillito (1973) considered that a division into primary and secondary aneurysm was justified and reviewed the 35 primary cases they were able to find in the literature, adding five of their own. They emphasised the need for surgery and also the frequency in the postoperative period of complications such as epilepsy, subdural haematoma/ hygroma, and hydrocephalus.

The present case shows that the diagnosis can be made by computed tomography and demonstrates the value of this technique in the postoperative management.

Address for reprint requests: Dr P. Macpherson, Division of Neuroradiology, Institute of Neurological Sciences, Southern General Hospital, Glasgow G51 4TF.

Accepted 9 March 1979

\section{Case report}

A male child was noted at birth to have a cardiac systolic bruit. Cardiomegaly, pulmonary hypertension, right ventricular hypertrophy, and patent ductus arteriosus were found, but there was no cardiac failure. The head circumference was $305 \mathrm{~mm}$, above the 90th centile. At that stage the fontanelle was soft but subsequently the hea became progressively larger and, when 16 week8 old, he was referred to this Institute for investio gation of hydrocephalus. His head circumference was $470 \mathrm{~mm}$, eye movements in an upward direc tion were impaired, and scalp veins were dilated.

\section{COMPUTED TOMOGRAPHY}

An EMI scan $(160 \times 160$ matrix $)$ demonstrated a sharply demarcated space-occupying lesion situated in the pineal region (Fig. 1). It projected into the bodies of the lateral ventricles, which were markedly dilated, and it also displaced laterally the trigone regions and occipital horns. The density was only slightly greater than brain, the average being 20 EMI units and the maximum 22 EMI units. Scans were repeated after $15 \mathrm{ml}$ of sodium iothalamate injection $70 \% \mathrm{w} / \mathrm{v}$ (Conray 420 ) were injected intravenously, and demonstrated a homogeneous increase in the density of the spaceoccupying lesion, which now had a posterior continuation to the region of the internal occipital protuberance (Fig. 2). The features were those of a large primary aneurysm of the vein of Galen with drainage via a dilated straight sinus into a widened torcula. The average density was now 40 EMI units and the maximum 52. On a low cut, feeding vessels were evident, two on the right and 


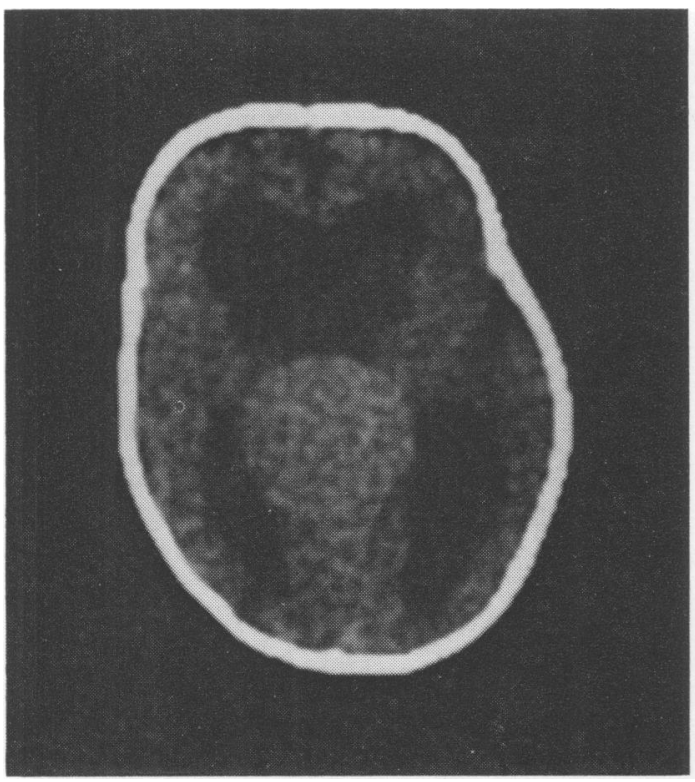

Fig. 1 Initial EMI scan showing space-occupying lesion in the pineal region and hydrocephalus.



Fig. 2 Enhanced scan demonstrating aneurysm of the vein of Galen with draining vessel.

three on the left (Fig. 3). There was a wide interhemispheric fissure and slight widening of the cerebral sulci.

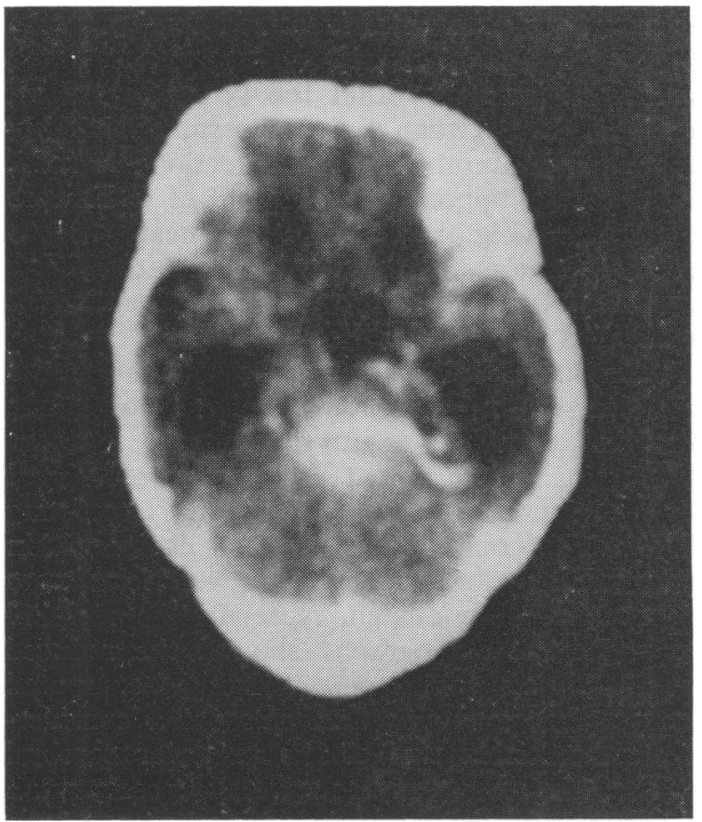

Fig. 3 Enhanced scan showing the feeding vessels. Two vessels loop round and enter the base of the aneurysm on the right and three lead to the aneurysm on the left.

\section{ANGIOGRAPHY}

Two large redundant vessels fed the aneurysm from the right posterior cerebral artery and three smaller ones from the left posterior cerebral artery. The internal carotid arteries contributed considerably to the supply via enlarged posterior communicating arteries.

\section{OPERATION}

The feeding vessels at the base of the aneurysm were occluded by either clipping or coagulation via a large right sided craniotomy (Matson, 1969). Two large vessels, which joined to form a common ampulla at the base of the aneurysm, were found on the right side and three smaller vessels on the left.

Frequent seizures occurred in the first 12 hours after operation and respiratory distress developed, so controlled mechanical ventilation was administered for three days.

An EMI scan on the third day after operation showed that the aneurysm was smaller. Blood clot was present, both within the sac and in the lateral ventricles. A cut at a higher level demonstrated a thin right subdural effusion but the examination excluded a complication requiring surgery. A scan one month later showed that there was still within 
the aneurysm an area of increased density (up to 45 EMI units) which was interpreted as clot. There was no longer clot within the ventricles, which were less dilated than before operation. Moderately sized bilateral subdural collections of fluid, whose density approximated to that of CSF, were now present (Fig. 4), indicating the need for subdural aspiration. Bilateral taps produced large quantities of fluid, and the effusions were drained externally for five days.

A further EMI scan two months after the operation showed that the subdural collections were then thin. The aneurysm was now isodense with brain. Enhancement produced a peripheral circle of increased density indicating circulating blood which communicated with a cavity within the resolving clot (Fig. 5). The ventricles were again rather more dilated, especially the occipital horns. Repeat angiography showed the reduced size of the aneurysm (less well than did computed tomography), and also demonstrated a considerable reduction in size of the posterior cerebral arteries. The aneurysm was supplied by some small branches from these vessels, which had not been visible on the original angiogram. Because the ventricles had increased in size, in spite of a free flow of contrast medium through the aqueduct during ventriculography, a ventriculoperitoneal

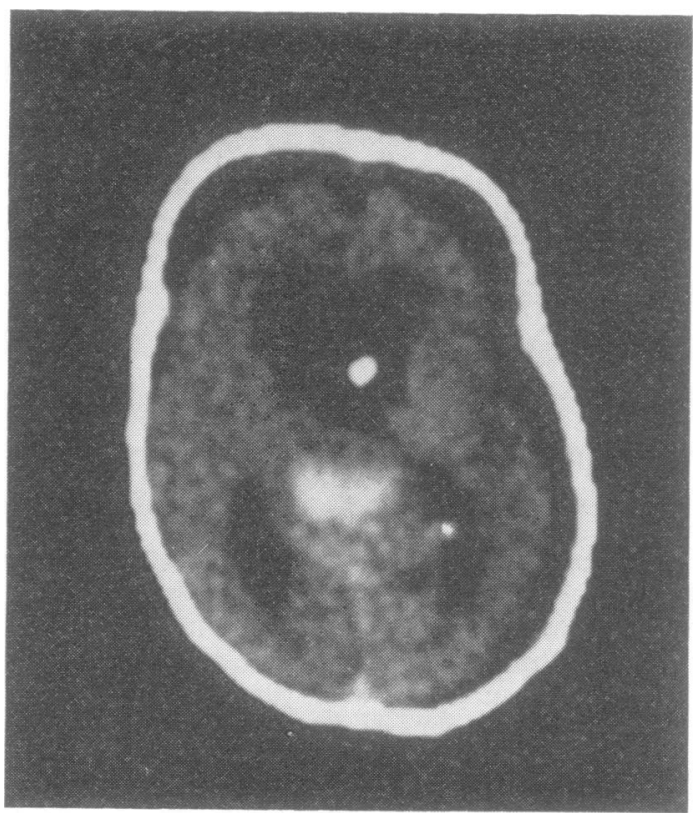

Fig. 4 Scan one month after operation. There is clot within the aneurysm, and bilateral subdural fluid collections are present.

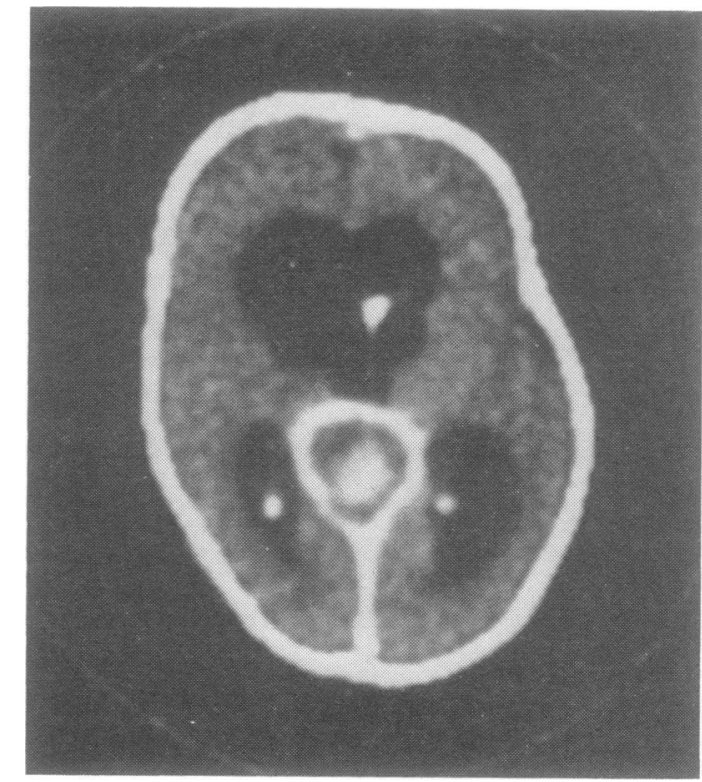

Fig. 5 Enhanced scan two months after operation. This shows that blood is circulating peripherally within the aneurysm which is now smaller. The central density which was not present on the unenhanced scan represents a cavity within the central clot connected toD the circulating blood. Subdural fluid collections are no longer present at this level. The intraventricular density is caused by the tip of a catheter which is attached to a reservoir.

shunt was inserted, and the child was discharged $\overrightarrow{+}$ home.

\section{FOLLOW-UP}

Computed tomography was repeated six months later. The ventricles were no longer dilated; the thin bilateral subdural effused collections were still present, their inner margins now showing slightly increased attenuation representing thickened membranes. Enhancement showed that within the contrast density in the aneurysm there was a small unenhanced area, interpreted as residual clot, but that the aneurysm was considerably smaller (Fig. 6). Measurement of comparable cuts showed that the diameter had reduced from an initial $62 \mathrm{~mm}$ to $20 \mathrm{~mm}$.

\section{Discussion}

Aneurysm of the vein of Galen can be diagnosed by computed tomography. The need for further studies depends upon what treatment is planned. Management of vein of Galen aneurysm varies according to the manner and time of presentation. When hydrocephalus is the main problem, as is 


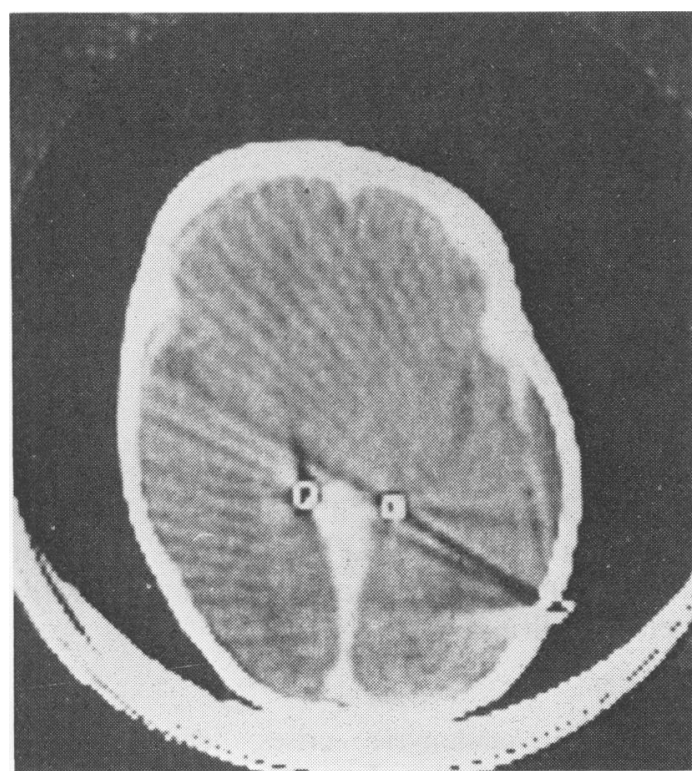

Fig. 6 Enhanced scan eight months after operation. There has been continued reduction in the size of the aneurysm. Ventricular dilatation is no longer present.

usual in older infants and in children (Amacher and Shillito, 1973), a simple CSF diversion may be carried out, in which case computed tomography will provide enough preoperative information and can be used to monitor subsequent progress. On the other hand, CSF diversion will neither relieve local distortion, which may be progressive, nor restore intracranial haemodynamics to normal: further arteriovenous shunting may cause cardiac failure in the neonatal period and later may interfere with cerebral perfusion. For these reasons, and also in order to prevent intracranial haemorrhage, obliteration of the arteriovenous fistula by operation is usually the preferred management (Yasargil et al., 1976). The surgeon will then wish for as much information as possible about the number and anatomy of the feeding vessels, and angiography will be necessary.

Although the aim of angiography is to demonstrate the feeding vessels, pooling of contrast medium in the sac of a large aneurysm and superimposition of large vessels can make visualisation of all feeding vessels very difficult. In the present case, computed tomography gave almost as much information about the large vessels as the preoperative angiogram, and neither investigation demonstrated the small feeding vessels responsible for persisting filling after operation.

With such a large aneurysm, access to its base can be difficult through the parietal craniotomy employed by Matson (1969) and still recommended by Amacher and Shillito (1973). Yasargil et al. (1976) prefer a posterior approach, with the patient sitting. Maravilla et al. (1978) have reported that computer reconstruction with sagittal and coronal scans can aid in localising the lesion precisely in relation to the tentorial incisura, and this may be helpful in planning the operation.

After operation we found computed tomography valuable in assessing changes in the size of the aneurysm and in demonstrating the development and absorption of the thrombosis within its cavity. It also assisted in the recognition and management of hydrocephalus and subdural effusions which Amacher and Shillito (1973) reported to be common complications of the operation. These authors also observed persistent filling of the aneurysm through small vessels which later thrombosed. Computed tomography seems preferable to angiography in following the changes in the aneurysm. Angiography is difficult to perform in small children and computed tomography has reduced its role considerably in the diagnosis and management of vein of Galen aneurysms.

\section{References}

Amacher, A. L., and Shillito, J. (1973). The syndromes and surgical treatment of aneurysms of the great vein of Galen. Journal of Neurosurgery, 39, 89-98.

Berger, P. E., Harwood-Nash, D. C., and Fitz, C. R. (1976). Computerised tomography in abnormal intracerebral collections of blood in children. Neuroradiology, 11, 29-33.

Hirano, A., and Terry, R. D. (1958). Aneurysm of the vein of Galen. Journal of Neuropathology and Experimental Neurology, 17, 424-429.

Maravilla, K. R., Kirks, D. R., and Maravilla, A. M. (1978). Computer reconstructed sagittal and coronal CT scans. Pediatric Radiology, 7, 65-69.

Matson, D. (1969). Neurosurgery of Infancy and Childhood, second edition, p. 772. Charles C. Thomas: Springfield, Illinois.

Milhorat, T. H. (1978). Paediatric Neurosurgery, p. 319. F. A. Davis: Philadelphia.

Morris, L. (1971). Pneumoencephalographic findings in a case of vein of Galen aneurysm. British Journal of Radiology, 44, 798-801.

O'Brien, M. S., and Schechter, M. M. (1970). Arteriovenous malformations involving the galenic system. American Journal of Roentgenology, 110, $50-55$.

Weisberg, L. A., Nice, C., and Katz, M. (1978). Cerebral Computed Tomography, p. 103. W. B. Saunders: Philadelphia, London, 'Toronto.

Yasargil, M. G., Antic, J., Lacka, R., Jain, K. K., and Boone, S. C. (1976). Arteriovenous malformations of vein of Galen: microsurgical treatment. Surgical Neurology, 6, 195-200. 